\title{
Pericardial tamponade caused by massive fluid resuscitation in a patient with pericardial effusion and end-stage renal disease -A case report-
}

\author{
Soonjae Hwang, Ji Young Bae, Tae-Wan Lim, In-Suk Kwak, and Kwang-Min Kim \\ Department of Anesthesiology and Pain Medicine, Hangang Sacred Heart Hospital, Seoul, Korea
}

Pericardial tamponade can lead to significant hemodynamic derangement including cardiac arrest. We experienced a case of pericardial tamponade in a patient with end-stage renal disease. Hemodynamic changes occurred by unexpectedly aggravated pericardial effusion during surgery for iatrogenic hemothorax. We quickly administered a large amount of fluids and blood products for massive bleeding and fluid deficit due to hemothorax. Pericardial effusion was worsened by massive fluid resuscitation, and thereby resulted in pericardial tamponade. Hemodynamic parameters improved just after pericardiocentesis, and the patient was transferred to the intensive care unit. (Korean J Anesthesiol 2013; 65: 71-76)

Key Words: End-stage renal disease, Hemodynamic change, Pericardial effusion, Pericardial tamponade.

Pericardial tamponade is a clinical syndrome leading to severe hemodynamic changes and causing pressure on the heart from fluid in the pericardial space [1]. Three major signs are low blood pressure, high central venous pressure, and tachycardia [2]. It requires a preemptive diagnosis and treatment due to high risk of mortality. The causes of pericardial effusion inducing pericardial tamponade are acute myocardial infarction, heart surgery, trauma, extensively spreading tumor, chest radiation, end-stage renal failure, invasive cardiac intervention, hypothyroidism, autoimmune disease, and acute inflammatory pericarditis [3]. Although 1-2 L of fluid retention may not generate any symptoms in chronic cases, pericardial tamponade could occur with just $150-250 \mathrm{ml}$ of fluid retention in acute process [4]. While the treatment is direct pericardiocentesis and intravascular volume expansion may be effective by administering fluid, the effect of fluid administration could be limited in some cases [5] and if excessive, it may aggravate pericardial tamponade [1].

The authors report a case that deteriorated to pericardial tamponade by massive fluid resuscitation in a patient who underwent emergent surgery due to hemothorax with massive bleeding.

Received: June 1, 2012. Revised: 1st, July 20, 2012; 2nd, August 8, 2012. Accepted: August 31, 2012.

Corresponding author: Tae-Wan Lim, M.D., Department of Anesthesiology and Pain Medicine, Hangang Sacred Heart Hospital, College of Medicine, Hallym University, 94-200, Yeongdeungpo-dong, Yeongdeungpo-gu, Seoul 150-030, Korea. Tel: 82-2-2639-5505, Fax: 82-2-2631-4387, E-mail: ltwhanzo@naver.com

(c) This is an open-access article distributed under the terms of the Creative Commons Attribution Non-Commercial License (http:// creativecommons.org/licenses/by-nc/3.0/), which permits unrestricted non-commercial use, distribution, and reproduction in any medium, provided the original work is properly cited. 


\section{Case Report}

A 73-year old male patient of $173 \mathrm{~cm}$ height and $52 \mathrm{~kg}$ weight visited hospital. The chief complaint was cellulitis in right ankle. He was under medication after being diagnosed with diabetes, hypertension, congestive heart failure, and left chronic tuberculous empyema ten years ago. He was undergoing hemodialysis three times a week after diagnosis of end-stage renal disease eight years previously. There were no specific findings during the hospital stay. He maintained normal blood pressure of $130 / 80 \mathrm{mmHg}$ and heart rate of $65-80$ beats per minute. Atrial fibrillation, normal left ventricular function, moderate concentric left ventricular hypertrophy, moderate pulmonary hypertension, severe tricuspid regurgitation, moderate

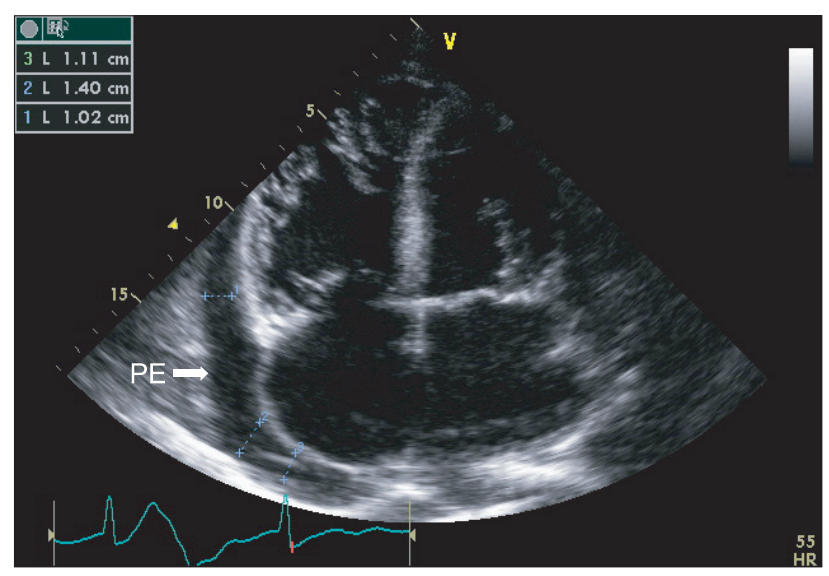

Fig. 1. Preoperative transthoracic echocardiography demonstrates moderate amount of pericardial effusion. PE: pericardial effusion.

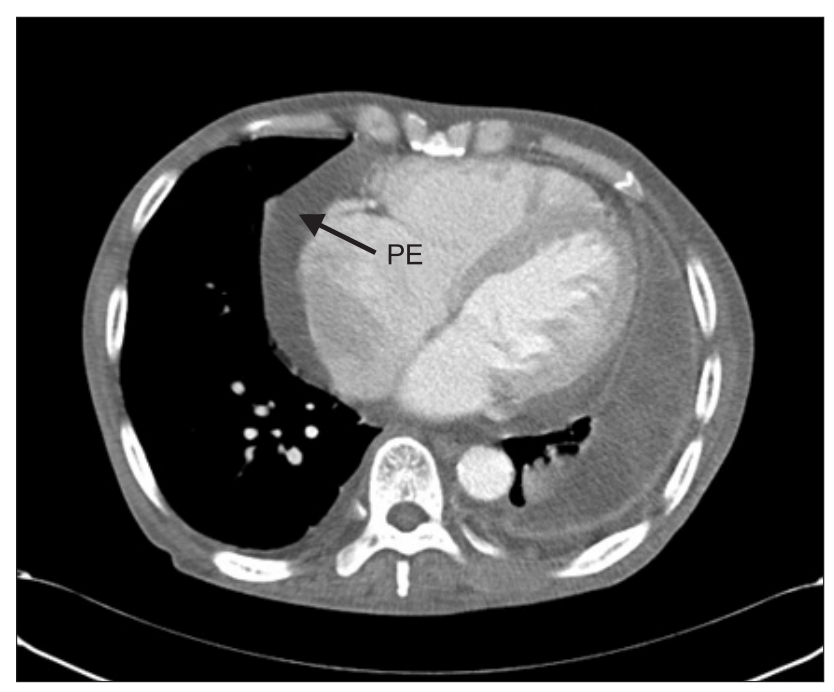

Fig. 2. Preoperative chest CT shows pericardial effusion. PE: pericardial effusion. mitral regurgitation, aortic regurgitation, and moderate pericardial effusion were observed in echocardiography (Fig. 1) performed during his hospital stay. Left lung empyema, right pleural effusion, pericardial effusion were confirmed by chest CT scans (Fig. 2) and simple chest X-rays (Fig. 3) and pulmonary function test showed a moderate restrictive pattern. Hemodialysis was performed the day before the operation and the result of the complete blood count before hemodialysis were hemoglobin level $8.8 \mathrm{~g} / \mathrm{dl}$.

On the fourth day of his in-hospital stay, sudden respiratory distress occurred after performing needle aspiration test of the right pleural effusion. Despite taking a simple chest X-ray immediately, our medical staff could not find any cause and observed him with oxygen therapy via nasal cannula. Respiratory distress got worse at a respiratory rate of 30 times per minute and an oxygen saturation level of $85 \%$ with changing mental status. Our staff inserted right chest tube 2 hours 30 minutes after performing needle aspiration biopsy. Approxi mately $1,500 \mathrm{ml}$ of blood was drained for about 10 minutes after chest tube insertion, with blood pressure of $90 / 40 \mathrm{mmHg}$, heart rate of 66 beats per minute, respiratory rate of 22 times per minute, and body temperature of $36.4^{\circ} \mathrm{C}$. Rapid fluid replacement with $1 \mathrm{~L}$ of normal saline via central venous catheter placed in the right internal jugular vein was performed and an emergency operation was decided upon. The patient's electronic medical record (EMR) was unidentifiable by anesthesiologists because of computer network error at that time. Hence, the emergency operation was began only by acknowledging that the patient with diabetes and hypertension was receiving hemodialysis and massive bleeding occurred during needle aspiration test at right pleura.

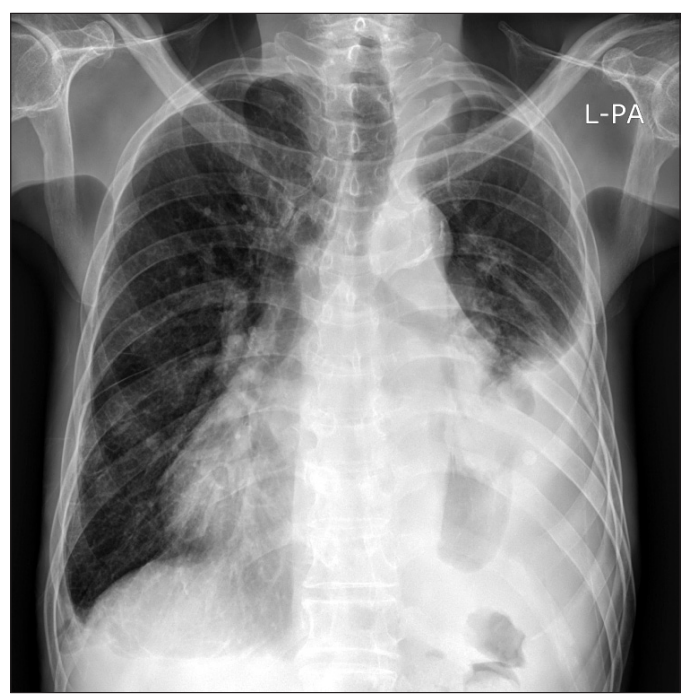

Fig. 3. Preoperative chest PA radiograph shows empyema (both hemithorax) and pericardial effusion. 
He was transferred to the operating room without any preoperative medication 20 minutes after chest tube insertion. At that time, his vital signs were blood pressure of $130 / 50$ $\mathrm{mmHg}$, heart rate of 60 beats per minute, respiratory rate of 22 times per minute, and body temperature of $34.9^{\circ} \mathrm{C}$. He was stuperous and his skin was cold but other physical examination could not be performed. Preoxygenation was attempted with $100 \%$ oxygen at $8 \mathrm{~L} / \mathrm{min}$ via mask and warm Voluven ${ }^{\circledR}(6 \%$ hydroxyethyl starch, Fresenius Kabi, Germany) was rapidly administered. In addition, non-invasive blood pressure, oxygen saturation, and electrocardiogram (ECG) were monitored. An arterial catheter for continuous hemodynamic monitoring was placed via the right femoral artery and a central venous catheter via the right femoral vein and $500 \mathrm{ml}$ of Voluven ${ }^{\circledR}$ was rapidly administered. After IV administration of $90 \mathrm{mg}$ ketamine and $50 \mathrm{mg}$ rocuronium for induction, endotracheal intubation was performed with a $35 \mathrm{Fr}$ left-sided double lumen endotracheal tube (Broncho-Cath ${ }^{\circledR}$, Mallinckrodt Medical Athlone, Ireland) and flexible fiberoptic bronchoscope (LF-GP, Olympus, Japan) was used for confirmation of proper placement. During lung ventilation, tidal volume of $100-120 \mathrm{ml}$, a peak inspiratory pressure of $40 \mathrm{cmH}_{2} \mathrm{O}$, blood pressure of 130/50 $\mathrm{mmHg}$, a heart rate of 65 beats per minute, and a central venous pressure of $17-18 \mathrm{mmHg}$ in supine position was checked. We attempted to maintain anesthesia with $1.5 \mathrm{~L} / \mathrm{min}$ of oxygen, $1.5 \mathrm{~L} / \mathrm{min}$ of nitrous oxide, and 2 vol\% of sevoflurane. However, inspired oxygen concentration was changed to $100 \%$, sevoflurane administration was stopped, and $1 \mathrm{mg}$ of midazolam was administered intravenously every 15 minutes after oxygen saturation fell to $84 \%$. After the patient was changed to leftsided lateral position, the location of tube was confirmed by a flexible fiberoptic bronchoscope with a central venous pressure of $21 \mathrm{mmHg}$. Left one-lung ventilation (OLV) was performed five minutes after changing the position to left-sided lateral position. Tidal volume and oxygen saturation decreased to

Table 1. Results of Arterial Blood Gas Analysis during General Anesthesia

\begin{tabular}{lccc}
\hline \multicolumn{1}{c}{ Variables } & Phase 1 & Phase 2 & Phase 3 \\
\hline $\mathrm{pH}$ & 7.276 & 7.073 & 7.124 \\
$\mathrm{PaCO}_{2}(\mathrm{~mm} \mathrm{Hg})$ & 69.5 & 103.2 & 90.3 \\
$\mathrm{PaO}_{2}(\mathrm{~mm} \mathrm{Hg})$ & 469.7 & 207.8 & 210.3 \\
$\mathrm{HCO}_{3}^{-}(\mathrm{mmol} / \mathrm{L})$ & 22.0 & 24.1 & 24.3 \\
$\mathrm{Base}^{2}$ excess $(\mathrm{mmol} / \mathrm{L})$ & -8.6 & -0.5 & -0.2 \\
$\mathrm{Hemoglobin}(\mathrm{g} / \mathrm{dl})$ & 5.8 & 5.8 & 5.8 \\
$\mathrm{Ca}^{2+}(\mathrm{mmol} / \mathrm{L})$ & 1.11 & 0.91 & 0.88 \\
$\mathrm{Na}^{+}(\mathrm{mmol} / \mathrm{L})$ & 141 & 141 & 143 \\
$\mathrm{~K}^{+}(\mathrm{mmol} / \mathrm{L})$ & 5.2 & 4.3 & 4.4 \\
$\mathrm{Cl}^{-}(\mathrm{mmol} / \mathrm{L})$ & 115 & 115 & 117 \\
$\mathrm{Glucose}(\mathrm{mg} / \mathrm{dl})$ & 139 & 145 & 126 \\
\hline
\end{tabular}

Phase 1: 25 minutes after start of surgery, Phase 2: 30 minutes after pericardial drainage, Phase 3: 10 minutes before end of surgery.
170-180 $\mathrm{ml}$ and $80 \%$ respectively. Peak inspiratory pressure increased up to $38 \mathrm{cmH}_{2} \mathrm{O}$.

Immediately on opening the right pleural cavity via right posterolateral thoracotomy, blood pressure decreased catastrophically and 1,500 $\mathrm{ml}$ of blood gushed out of the pleural cavity followed by about additional $1,200 \mathrm{ml}$ of blood loss. Arterial blood gas analysis was performed 25 minutes after the beginning of surgery (Table 1). Subsequently, 3 L of Voluven ${ }^{\circledR}$ and $1 \mathrm{~L}$ of normal saline were administered rapidly during the half an hour after the beginning of surgery. At half an hour after the beginning of surgery, our hospital computer network was restored; packed red blood cells and fresh frozen plasma were administered. Then central venous pressure increased up to 38 mmHg with decreased active bleeding, fluid administration was minimized for concern about volume overload. Oxygen saturation level decreased continuously even though peak inspiratory pressure was above $40 \mathrm{cmH}_{2} \mathrm{O}$ during one-lung ventilation. One-lung and two-lung ventilation were performed in turn along with manual and mechanical ventilation by maintaining tidal volume of $120 \mathrm{ml}$, respiratory rate of $60-$ 70 times per minute, peak inspiratory pressure of $22 \mathrm{cmH}_{2} \mathrm{O}$, inhaled oxygen concentration of $100 \%$, and oxygen saturation of 91-93\%. Although fluid administration was minimized, central venous pressure increased up to $50 \mathrm{mmHg}$, blood pressure and heart rate were maintained at $120 / 60 \mathrm{mmHg}$ and 67 beats per minute respectively and there were no abnormal ECG finding. Initial blood loss via chest tube before surgery was $1,500 \mathrm{ml}$, estimated blood loss during operation was 2,700 $\mathrm{ml}$ for 40 minutes and there was no urine output. The patient was administered $4,000 \mathrm{ml}$ of Voluven ${ }^{\circledR}, 2,000 \mathrm{ml}$ of normal saline, 5 pints of packed RBCs, and 5 pints of fresh frozen plasma. At 45 minutes after the beginning of surgery, central venous pressure suddenly increased up to $58 \mathrm{mmHg}$ with falling in blood pressure of $70 / 40 \mathrm{mmHg}$, bradycardia of 25-30 beats per minute. $0.5 \mathrm{mg}$ of atropine and $0.5 \mathrm{mg}$ of epinephrine were administered immediately through a central line and the pericardium was checked by the surgeon for suspected pericardial tamponade while resuscitation was being prepared. The surgeon confirmed pericardial expansion and made a pericardial window immediately. After approximately $800 \mathrm{ml}$ of yellow serous fluid with no blood was drained from the pericardial space, vital signs stabilized with recovering heart rate 60 beats per minute. Thereafter, blood pressure of 115/50 $\mathrm{mmHg}$ and heart rate of 65 beats per minute were maintained and central venous pressure was reduced to $45 \mathrm{mmHg}$. To maintain blood pressure, $20 \mathrm{mg}$ of ephedrine and $1 \mathrm{~g}$ of calcium gluconate were administered divided. Arterial blood gas analysis was performed 30 minutes after pericardial window (Table 1). Although no active bleeding focus was found in the surgical field, blood leak continued under the damaged diaphragm. It 
was decided to observe the patient in the intensive care unit after primary closure. Flexible fiberoptic bronchoscopy was performed postoperatively and a small fresh blood clot was detected at the right bronchus. The patient was transferred to the ICU while maintaining a double lumen endotracheal tube from concern about failure of finding the bleeding focus and the risk of rebleeding. The total amount of fluid administrated during surgery was $4,100 \mathrm{ml}$ of Voluven ${ }^{\circledR}, 3,000 \mathrm{ml}$ of normal saline, 7 pints of packed RBCs, and 5 pints of fresh frozen plasma. Blood loss before surgery was $1,500 \mathrm{ml}$ and intraoperative blood loss was $2,700 \mathrm{ml}$. Upon arrival on the ICU, the patient was stable with blood pressure of $97 / 72 \mathrm{mmHg}$, heart rate of 74 beats per minute and a tympanic membrane temperature of $35.7^{\circ} \mathrm{C}$. No pulmonary edema was observed on postoperative chest X-ray (Fig. 4). Hemoperitoneum was confirmed on abdominal CT scan but we decided to observe carefully because there was no active bleeding focus in the abdominal cavity on the celiac artery angiogram. The patient exhibited stable vital signs after continuous renal replacement therapy during follow-up period and about $900 \mathrm{ml}$ of bleeding via chest tube occurred on the first postoperative day. However, the chest tube was removed and hemoperitoneum was resolved as bleeding diminished 10 days postoperatively. Moderate pericardial effusion was detected again on echocardiography. The patient is currently admitted to the intensive care unit under mechanical ventilation due to respiratory failure.

\section{Discussion}

Pericardial effusion is commonly detected clinically and patients have to be suspected of pericardial effusion when

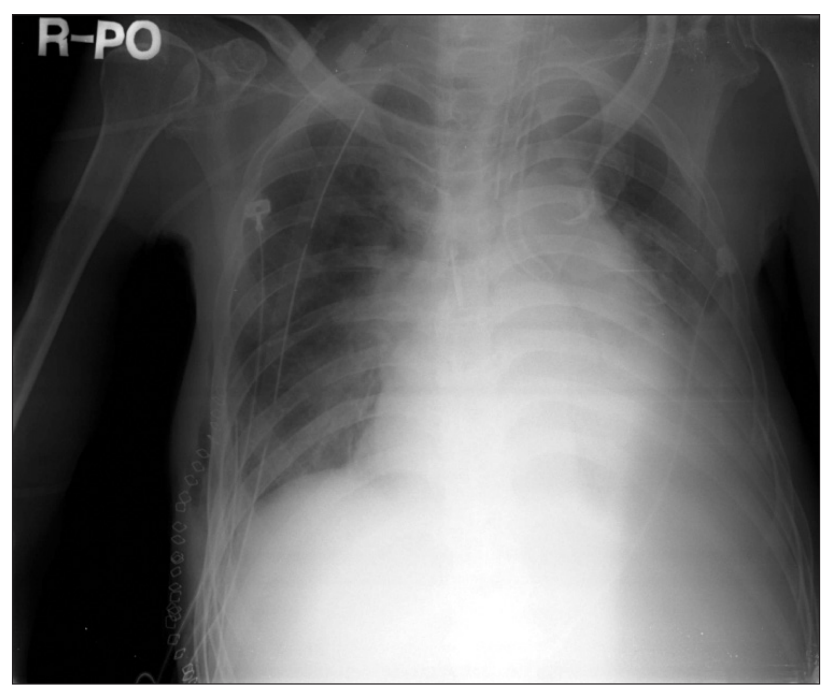

Fig. 4. Postoperative chest AP radiograph shows empyema (left hemithorax), right pleural effusion, and pericardial effusion. they develop cardiomegaly despite normal lung field on simple X-ray [3]. To find causes of pericardial effusion from the beginning is not easy. The causes need to be identified by relating the underlying diseases of patients or procedures history. According to Corey et al. [6], the leading causes are malignant tumor, virus infection, radiation exposure induced inflammation, collagen vascular disease, and uremia. Patients with end-stage renal disease, in particular, are more likely to develop chronic pericardial effusion due to continuous volume overload [7]. In such cases, pericardial tamponade may be resulted when the volume of pericardial effusion rapidly increases, without exhibiting hemodynamic symptoms. For this reason, anesthetic management may be challenging due to unexpected drastic hemodynamic changes as shown in this case where the operation was performed without echocardiographic detection of pericardial effusion. When pericardial effusion slowly develops, intrathoracic pressure gradually increases due to stretched parietal pericardium and the heart endures to some extent although 1-2 L of fluid is accumulated. However, pericardial tamponade may result from drastically increased intrathoracic pressure when pericardial effusion rapidly develops [8]. Therefore, prompt diagnosis and treatment need to be immediately implemented when pericardial tamponade is suspected [9].

When pericardial effusion generates pericardial tamponade inducing drastic and hemodynamic deterioration, dyspnea, tachycardia, jugular venous distension, and pulsus paradox are observed and these symptoms eventually lead to hypotension and shock [3]. The classic triad (Beck's triad) of pericardial tamponade are hypotension, increased central venous pressure, and tachycardia. However, appropriate caution should be taken since not a few patients develop sudden cardiovascular collapse without showing apparent signs [10]. While diagnosing the causes of drastic hypotension in this case, pericardial distension was identified with suspicion of pericardial tamponade. However, pericardial tamponade was hardly suspected in this case just before the incident of hypotension since central venous pressure alone increased with normal blood pressure and heart rate. In addition, increased central venous pressure was thought to have resulted from rapid, massive fluid resuscitation for treating preoperative and intraoperative massive bleeding induced hypovolemia. However, increased central venous pressure was attributable to prolonged excessive volume overload in the heart due to severe tricuspid regurgitation, moderate pulmonary artery hypertension and congestive heart failure despite alleviated pericardial tamponade. Although hypotension and increased central venous pressure coincided with the Beck's triad of pericardial tamponade, bradycardia of 20-30 beats per minute gave difficulty in distinguishing pericardial tamponade from other heart problems. When 
uremia developed due to chronic renal failure as this case or hypothyroidism exists, special attention should be paid to patient's status without tachycardia [11]. Factors affecting the severity of pericardial tamponade include pericardial compliance, central venous pressure, acuity, the amount of pericardial fluid, and the speed of fluid accumulation. Low central venous pressure, high acuity, massive amount of pericardial fluid and low pericardial compliance are more likely to lead to cardiovascular collapse [1].

Echocardiography as well as CT scan and MRI are widely used to diagnose pericardial effusion and tamponade [3]. Considering the possibility of pericardial tamponade is not easy when hemodynamic change is induced by intraoperative pericardial tamponade, especially in the cases of preoperatively undiagnosed pericardial effusion or proceeding surgery with underestimated severity of pericardial effusion. Although pericardial effusion was directly detected by the surgeons in the surgical view of the opened chest in this case, diagnosis is hard in operations other than thoracic surgery. Therefore, transesophageal echocardiography (TEE) is beneficial in differential diagnosis and treatment of associated diseases when drastic intraoperative hemodynamic changes occur [12]. However, TEE could not be performed in this study since our hospital was not equipped with TEE.

Anesthetic management of pericardial effusion and tamponade requires thorough review of the pre-anesthetic evaluation and anesthetic methods [8]. In this case report, examining the medical history and laboratory results of the patient was problematic due to the nature of emergency surgery and a computer network error. Although the surgeons were aware of the fact that the patient was receiving hemodialysis due to end-stage renal failure, we were unable to take into account the possibility of pericardial tamponade induced by pericardial effusion due to more urgent massive intrathoracic bleeding. Moreover, it is critical to prevent interference with venous return due to increased airway pressure during positive pressure ventilation [8]. In this case, $\mathrm{PaCO}_{2}$ reached excessively high levels because adequate ventilation was not performed due to high airway pressure during OLV and underlying pulmonary diseases (Table 1). Although TLV and manual ventilation were concurrently performed together with OLV under mechanical ventilation, respiratory acidosis was not corrected. Moreover, sustained high airway pressure might have influenced venous return adversely. Cardiac structures enlarged by massive pericardial effusion and congestive heart failure were expected to affect the elevated airway pressure due to compressed dependent lung in lateral position. This was inferred from the fact that airway pressure and peak inspiratory pressure became normalized in supine position upon the completion of surgery. For this reason, there was very little possibility of bronchospasm.
Arterial cannulation is essential and central venous cannulation is also necessary when considering potential massive bleeding. However, pericardiocentesis should not be delayed due to placement of central venous catheter. Fluids, blood products, vasopressor, and other medications should be prepared and potential arrhythmia that may develop while manipulating the pericardium and heart should be dealt with by defibrillation [8]. An elevated preload should be sustained during aesthesia and maintaining normal heart rate, afterload, contractility, and sinus rhythm is crucial $[5,8]$.

Although the treatment of choice of pericardial tamponade is pericardiocentesis, there are some cases where the procedure cannot be immediately implemented. Hence, fluids are administered aiming for expanding intravascular volume as a temporary treatment to increase arterial pressure and cardiac index [5]. However, the patient in this study suddenly exhibited increased central venous pressure with decreased blood pressure and drastically deteriorating pericardial tamponade intraoperatively. This was attributable to a sudden decrease in cardiac output due to increased pressure in the pericardium resulting from pericardial exudation as a large volume of fluids was administered in a short period of time. Moreover, pericardial exudation was thought to be worsened because 1 liter of crystalloid was rapidly injected against bleeding after fine needle aspiration biopsy. This assumption is based on the change that the patient had moderate pericardial effusion preoperatively but $800 \mathrm{ml}$ of effusion fluid was drained during operation. A study reported that pericardial tamponade induced by massive fluid resuscitation developed with clear serous fluid accumulation in the pericardial cavity as in this case report [1]. A 37-year old female patient without any underlying diseases was treated following a car accident with pelvic fracture, liver and spleen rupture, and mediastinal hemorrhage. After large volumes of fluids were administered, pulseless electrical activity and shock developed due to pericardial effusion leading to tamponade. However, her status recovered immediately after drainage of pericardial effusion. The study reported that the incident of pericardial effusion without intrathoracic injury resulted from the movement of intravascular fluid to interstitial space due to acute capillary leak affected by massive fluid resuscitation, multiple trauma, severe bleeding, reperfusion, and a prolonged anesthetic period [1]. In this case, we pointed out that decreased osmotic pressure resulted from a drop in albumin levels in chronic renal failure as an additional mechanism. However, the expected pulmonary edema did not occur. Pressed left heart and increased left ventricular end-diastolic pressure resulted from an increase in the volume of right heart in the limited pericardial space caused by massive fluid resuscitation. Consequently, the blood pressure might be lowered due to decreased cardiac output [5]. Aggravated pulmonary artery 
hypertension and severe tricuspid regurgitation induced by hypercapnia and acidosis could be involved in increasing the volume of right heart. Cardiac function might be weakened due to right heart volume overload leading to myocardial ischemia. Moreover, the declined cardiac contractility developed by worsened myocardial ischemia resulted from hypocalcemia, severe acidosis, and congestive heart failure is also thought to be involved in decreased blood pressure. Airway management should be conducted promptly, since the prolonged condition with low oxygen saturation after performing fine needle aspiration biopsy in pleural cavity is thought to have an adverse effect.
In conclusion, the risk of pericardial effusion or pericardial tamponade should be identified by considering the underling illnesses of patient during pre-anesthetic evaluation. A thorough pre-anesthetic evaluation is particularly crucial in patients with end-stage renal disease since they are highly likely to be at risk of pericardial effusion. In such cases, hemodynamic changes should be carefully monitored while taking potential intraoperative pericardial tamponade into account. In addition, transesophageal echocardiography is recommended for an accurate diagnosis. Pericardial effusion and tamponade should be considered carefully, especially when administering excessive volumes of fluids or blood products before the pericardiocentesis.

\section{References}

1. Hashim R, Frankel H, Tandon M, Rabinovici R. Fluid resuscitation-induced cardiac tamponade. J Trauma 2002; 53: 1183-4.

2. Aldridge HE, Jay AW. Central venous catheters and heart perforation. CMAJ 1986; 135: 1082-4.

3. Sagrista-Sauleda J, Merce AS, Soler-Soler J. Diagnosis and management of pericardial effusion. World J Cardiol 2011; 3: 135-43.

4. Levitt MA, Cunningham JD, Curtiss SI, Brower ST. Intraoperative cardiac tamponade complicating esophagogastrectomy. J Cardiovasc Surg (Torino) 1998; 39: 245-7.

5. Sagrista-Sauleda J, Angel J, Sambola A, Permanyer-Miralda G. Hemodynamic effects of volume expansion in patients with cardiac tamponade. Circulation 2008; 117: 1545-9.

6. Corey GR, Campbell PT, Van Trigt P, Kenney RT, O'Connor CM, Sheikh KH, et al. Etiology of large pericardial effusions. Am J Med 1993; 95: 209-13.

7. Kleiman JH, Motta J, London E, Pennell JP, Popp RL. Pericardial effusions in patients with end-stage renal disease. Br Heart J 1978; 40: 190-3.

8. Grocott HP, Gulati H, Srinathan S, Mackensen GB. Anesthesia and the patient with pericardial disease. Can J Anaesth 2011; 58: 952-66.

9. Markiewicz W, Borovik R, Ecker S. Cardiac tamponade in medical patients: treatment and prognosis in the echocardiographic era. Am Heart J 1986; 111: 1138-42.

10. Nasim A, Cooper GG, Ah-See AK. Cardiac tamponade due to central venous catheterization. J R Coll Surg Edinb 1992; 37: 337-9.

11. Spodick DH. Acute cardiac tamponade. N Engl J Med 2003; 349: 684-90.

12. Ng A, Swanevelder J. Perioperative echocardiography for non-cardiac surgery: what is its role in routine haemodynamic monitoring? Br J Anaesth 2009; 102: 731-4 